\author{
A Test Set of Real-world Mixed \\ Integer Programming Problems ${ }^{1}$ \\ Robert E. Bixby \\ E. Andrew Boyd \\ Ronni R. Indovina \\ November, 1991 \\ (revised January 1992)
}

TR91-38

\footnotetext{
${ }^{1}$ The preparation of this manuscript was supported in part by NSF grant CCR-8815914 and AFOSR grant AFOSR-90-0273, both to Rice University.
} 



\title{
MIPLIB:
}

\section{A Test Set of Real-world Mixed Integer Programming Problems}

\author{
Robert E. Bixby E. Andrew Boyd Ronni R. Indovina
}

November, 1991

\section{Introduction}

Integer programming problems, both pure and mixed, have traditionally been considered very difficult. Indeed, there are currently no guaranteed polynomial-time algorithms available for general integer programming. Within the last ten years, however, major advances in preprocessing methods and cutting plane techniques have provided more insight into what makes these problems so difficult. These breakthroughs have sparked much interest and have led to new research in the field of integer programming.

Integer programs are problems that consist of maximizing or minimizing a linear function on a linearly defined constraint set with some or all of the variables restricted to take on integer values. If only some of the variables have this restriction, the problem is a mixed integer program. These problems have applications in many diverse areas. Among these applications are: fiber optic network design, modeling the acquisition, manufacturing and distribution of natural gas, machine and job scheduling, vehicle routing, drainage system design, generator scheduling, airline crew and flight scheduling, modeling automobile acquisition and selling. This wide applicability of integer programming presents a real need for efficient methods for solving both pure and mixed integer programming problems. One hindrance to research in this area has been a lack of general availability of real models.

In 1985, David M. Gay introduced a library of linear programming problems [4] that is electronically accessible through NETLIB [3], and, in 1990, Gerhard Reinelt in- 
troduced TSPLIB, an electronically accessible library of Traveling Salesman problems [6]. The availability of real models through both of these libraries has sparked much computational research on linear programming and traveling salesman problems. In fact, since the creation of TSPLIB, 13 problems that were previously considered "unsolvable" have been solved to provable optimality. Given that these two libraries have had such an impact on their respective fields, we felt that a similar effort was needed in the area of integer programming. MIPLIB is an electronic library of both pure and mixed integer programs created in an effort to make real models available to researchers working on such problems.

The types of problems included in the MIPLIB set are varied. For every application of integer programming mentioned above, there is at least one model of that type in the library. In addition, the level of difficulty of the models in the library is equally as varied. The bell series of problems is a set of relatively small but incredibly difficult models, arising from the design of fiber optic networks, which are characterized by a large number of general integer variables. The only method that has been able to solve these is a new code developed by Cook, Rutherford, Scarf, and Shallcross [1] based on the basis reduction technique of Scarf and Lovasz [5]. The pxxxx problems are also difficult problems for a standard branch and bound approach. A preprocessing and cutting plane technique developed by Crowder, Johnson, and Padberg [2] has been very successful in dealing with these models. The airxx models are all set partitioning problems that arise in airline crew scheduling. These airline models are generally very difficult, and in particular, no optimal solution has yet been proven for air05. The stein models, while all very small, are not as harmless as they appear, particularly stein45. Another very difficult model is rentacar, which models automobile acquisition and selling. The basis matrix suddenly becomes very dense during the solution process, causing basis factorizations to slow down and potential memory difficulties. The problem misc07 also seems to be very difficult.

\section{Index}

The following is a two-part index of the models currently contained in the library. The first part contains statistics for each problem, and the second part contains information regarding the origins of each problem. In PART A, the first column, NAME, 
contains the name of the model. The next two columns, ROWS and COLS, contain the number of rows in the constraint matrix, not including free rows, and the number of variables in the problem, respectively. The column INT tells the number of variables that are restricted to integer values, and the column $0 / 1$ tells how many of these integer variables are binary. The last two columns report the optimal solution value and the optimal solution value with the integrality restrictions relaxed, respectively. In the column INT SOLUTION however, the solutions reported are followed by qualifiers. The qualifier (opt) indicates that the reported solution is indeed integer optimal, while (not opt) indicates that the reported solution is not integer optimal. The qualifier (opt)* indicates that the solution is reportedly integer optimal, as stated by various sources, but it has not yet been verified by us.

The first column of PART B is, again, NAME. The second column, ORIGINATOR, gives the name of the person or institution with which the problem originated. The next column, FORMULATOR, gives the name of the person or organization responsible for formulating the model, and the last column, DONATOR, gives the name of the person or institution who contributed the problem. 


\section{PART A : STATISTICS}

\begin{tabular}{|c|c|c|c|c|c|c|}
\hline NAME & ROWS & CŌLS & INT & $0 / 1$ & INT SOLUTION & LP SOLŪTION \\
\hline air01 & 23 & 771 & 771 & ALL & 6796 (opt) & 6743.0 \\
\hline air02 & 50 & 6774 & 6774 & ALL & 7810 (opt) & 7640.0 \\
\hline air03 & 124 & 10757 & 10757 & ALL & 340160 (opt) & 338864.25 \\
\hline air04 & 823 & 8904 & 8904 & ALL & $56138(\mathrm{opt})^{*}$ & 55535.436 \\
\hline air05 & 426 & 7195 & 7195 & ALL & $26402($ not opt) & 25877.609 \\
\hline air06 & 825 & 8627 & 8627 & ALL & 49649 (opt) & 49616.364 \\
\hline bell3a & 123 & 133 & 71 & 39 & 878430.32 (opt)* & 862578.64 \\
\hline bell3b & 123 & 133 & 71 & 39 & $11786160.62(\mathrm{opt})^{*}$ & 11404143.89 \\
\hline bell4 & 105 & 117 & B4 & 34 & $18541484.20(\text { opt })^{*}$ & 17984775.91 \\
\hline bell5 & 81 & 104 & 58 & 30 & $8966406.49(\text { opt })^{*}$ & 8608417.95 \\
\hline bm23 & 20 & 27 & 27 & ALL & $34(o p t)$ & 20.57 \\
\hline cracpb1 & 143 & 572 & 572 & ALL & 22199 (opt) & 22199.0 \\
\hline diamond & 4 & 2 & 2 & ALL & integer infeasible & -1.0 \\
\hline dsbmip & 1182 & 1886 & 192 & 160 & $-305.198(\mathrm{opt})$ & -305.198 \\
\hline egout & 98 & 141 & 55 & ALL & $568.101(\mathrm{opt})^{*}$ & 149.589 \\
\hline enigma & 21 & 100 & 100 & ALL & $0.0(0 p t)$ & 0.0 \\
\hline fixnet3 & 478 & 878 & 378 & ALL & $51973(\text { opt } t)^{*}$ & 40717.018 \\
\hline flugpl & 18 & 18 & 11 & $\mathbf{0}$ & 1201500 (opt) & 1167185.73 \\
\hline gen & 780 & 870 & 150 & 144 & 112313 (opt) & 112130.0 \\
\hline khb05250 & 101 & 1350 & 24 & ALL & 106940226 (opt) & 95919464.0 \\
\hline 1152lav & 97 & 1989 & 1989 & ALL & 4750 (not opt) & 4656.36 \\
\hline lp4l & 85 & 1086 & 1086 & ALL & 2967 (opt) & 2942.5 \\
\hline lseu & 28 & 89 & 89 & ALL & 1120 (opt) & 834.68 \\
\hline modglob & 291 & 422 & 98 & $A L L$ & $20740508(\text { opt })^{*}$ & 20430947.0 \\
\hline $\operatorname{misc01}$ & 54 & 83 & 82 & $A L L$ & 563.5 (opt) & 57.0 \\
\hline misc02 & 39 & 59 & 58 & $A L L$ & 1690 (opt) & 1010.0 \\
\hline misc03 & 96 & 160 & 159 & ALL & $3360(\mathrm{opt})$ & 1910.0 \\
\hline misc04 & 1725 & 4897 & 30 & ALL & 2666.699 (opt) & 2656.42 \\
\hline misco5 & 300 & 136 & 74 & ALL & 2984.5 (opt) & 2930.9 \\
\hline miвc06 & 820 & 1808 & 112 & ALL & 12850.8607 (opt) & 12841.69 \\
\hline misc07 & 212 & 260 & 259 & $A L L$ & 2810 (not opt) & 1415.0 \\
\hline $\bmod 008$ & 6 & 319 & 319 & ALL & 307 (opt) & 290.93 \\
\hline $\bmod 010$ & 146 & 2655 & 2655 & ALL & $6548(o p t)$ & 6532.08 \\
\hline $\bmod 011$ & 4480 & 10958 & 96 & ALL & $-54558535(\mathrm{opt})^{*}$ & -62121982.552 \\
\hline $\bmod 013$ & 62 & 96 & 48 & ALL & 280.95 (opt) & 256.02 \\
\hline noswot & 182 & 128 & 100 & 75 & -43 (opt) & -43.0 \\
\hline p0033 & 16 & 33 & 33 & ALL & 3089 (opt) & 2520.57 \\
\hline p0040 & 23 & 40 & 40 & ALL & 62027 (opt) & 61796.55 \\
\hline p0201 & 133 & 201 & 201 & ALL & 7615 (opt) & 6875.0 \\
\hline p0282 & 241 & 282 & 282 & ALL & 258411 (opt) & 176867.50 \\
\hline p0291 & 252 & 291 & 291 & ALL & 5223.7490 (opt) & 1705.13 \\
\hline p0548 & 176 & 548 & 548 & ALL & $8691(\mathrm{opt})$ & 315.29 \\
\hline p2756 & 755 & 2756 & 2756 & ALL & $3124(o p t)^{*}$ & 2688.75 \\
\hline pipex & 25 & 48 & 48 & ALL & 788.263 (opt) & 773.751 \\
\hline rentacar & 6803 & 9557 & 55 & ALL & 30356761 (opt)* & 28806137.644 \\
\hline rgn & 24 & 180 & 100 & ALL & 82.1999 (opt) & 48.7999 \\
\hline sample2 & 45 & 67 & 21 & $A L L$ & 375 (opt) & 247.0 \\
\hline sentoy & 30 & 60 & 60 & ALL & -7772 (opt) & -7839.278 \\
\hline stein 15 & 36 & 15 & 15 & ALL & $9(\mathrm{opt})$ & 7.0 \\
\hline stein 27 & 118 & 27 & 27 & ALL & 18 (opt) & 13.0 \\
\hline stein 45 & 331 & 45 & 45 & ALL & $30(\text { opt })^{*}$ & 22.0 \\
\hline steing & 13 & 9 & 9 & ALL & 5 (opt) & 4.0 \\
\hline vpm1 & 234 & 378 & 188 & ALL & $20(\mathrm{opt})^{*}$ & 15.4167 \\
\hline
\end{tabular}




\section{PART B : ORIGINS}

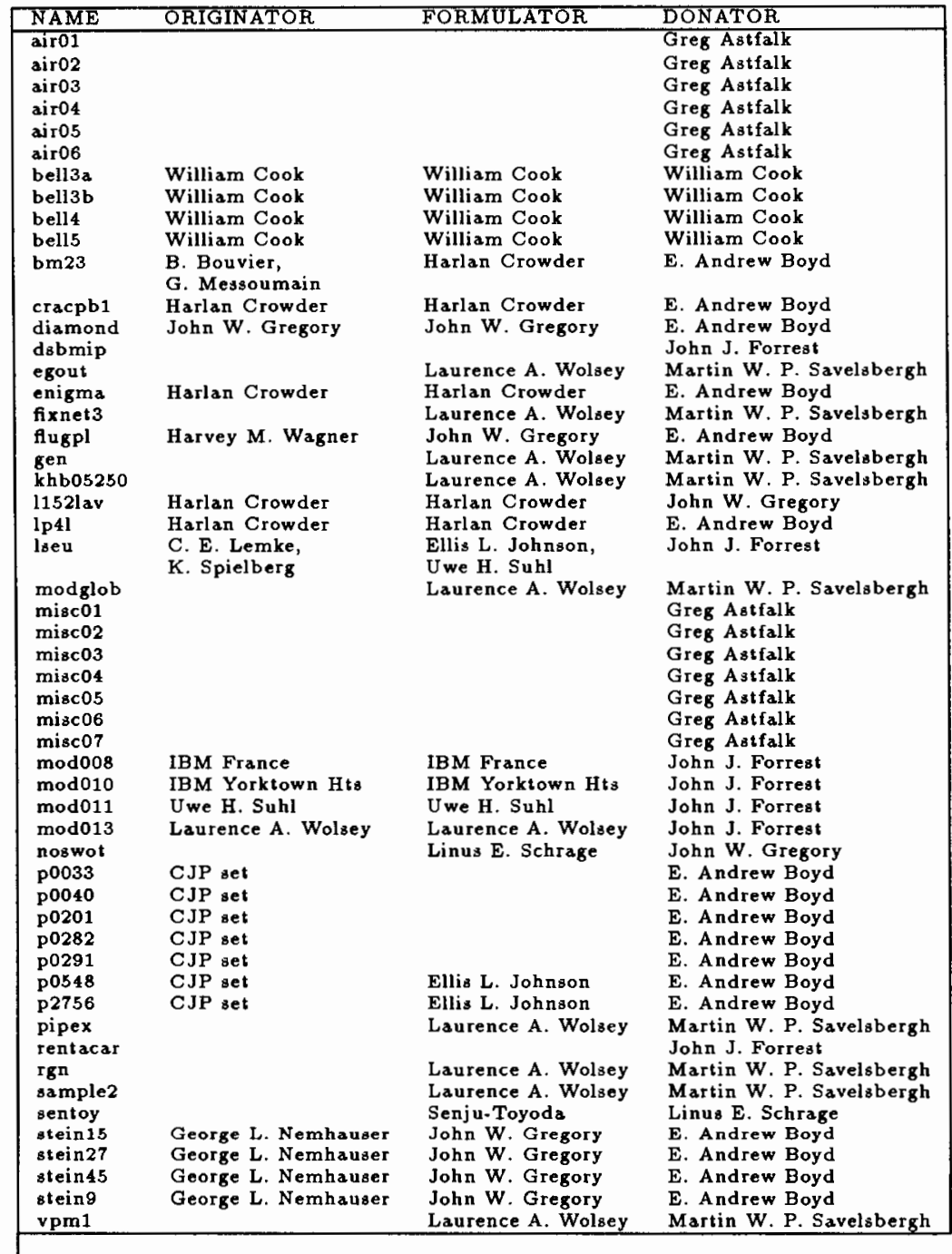




\section{MIPLIB Specifics}

Each problem in MIPLIB is stored in a separate file. Each file begins with a header section, followed by the actual problem data, which is stored in MPS format ${ }^{1}$. Each line of the header section has an $*$ in column one, and no lines are skipped between the header and the data. The following is a description of the header section:

*NAME: 1 line. This section contains the name of the problem.

*ROWS: 1 line. This section contains the number of constraints in the problem, not including free rows.

*COLUMNS: 1 line. This section contains the total number of variables in the problem.

*INTEGER: 1 line. This section contains the number of variables that are restricted to integer values.

*NONZERO: 1 line. This section contains the number of nonzeroes in the constraint matrix, not including free rows.

*BEST SOLN: 1 line. This section contains the best known solution to the problem. If the solution given is optimal, then the indicator (opt) will appear behind the solution. It is also possible that either "infeasible" or "integer infeasible" will appear here, which are self-explanatory terms.

*LP SOLN: 1 line. This section contains the optimal solution to the linear relaxation of the problem.

*SOURCE: 3 lines. The first line contains the name of the person (or organization) responsible for originating the problem. The second line contains the name of the person (or organization) responsible for formulating the problem as a MIP. Following this, in parentheses, is the name of the institution with which the person is affiliated. The third line contains the name of the person who donated the problem, along with the name of his or her affiliated institution in

\footnotetext{
${ }^{1}$ For detailed documentation on MPS format, see an IBM MPSX manual.
} 
parentheses. If any line is blank, it is because that information is not available. (Each line will have an asterisk in column 1.)

*APPLICATION: 1 line. This section contains a brief description of the application from which the problem arose. It is possible that "unknown" will appear here, which is self-explanatory.

*COMMENTS: 3 lines. This section will contain other miscellaneous information about the problem. Things that may appear here are data pertaining to the number of binary integer variables in the problem, constraint classifications, comments as to the difficulty of the problem, etc. Also, if the optimal solution reported by the contributor could not be verified, the system with which this solution was obtained is reported here. (Each line will have an asterisk in column 1.)

The section labels appear exactly as they do here, and no lines are skipped between sections. All data for the various sections begin in column 16 .

\section{Accessing MIPLIB}

The library is accessible through SOFTLIB, an electronic software distribution system available through Internet. By sending e-mail to softlib@rice.edu with a message body reading "send catalogue," you will receive via electronic mail a list of software codes and libraries currently available for public access through the system, along with specific instructions for accessing each one.

In addition to making this library available for public use, we wish to invite comments and suggestions regarding the library. Contributions of new problems to the library would also be greatly appreciated, as well as any new solutions that have been proven optimal.

To make submissions or comments, please contact Dr. Robert E. Bixby or Dr. E. Andrew Boyd, Department of Mathematical Sciences, Rice University, Houston, TX 77251 , or send e-mail to either bixby@rice.edu or boyd@rice.edu. 


\section{References}

[1] William Cook, Thomas Rutherford, Herbert Scarf, and David Shallcross, "An implementation of the generalized basis reduction algorithm for integer programming," Cowles Foundation Discussion Paper No. 990, 1991, Yale University, New Haven, Connecticut.

[2] H. Crowder, E. L. Johnson, and M. Padberg, "Solving large-scale zero-one linear programming problems," Operations Research 31 (1983), 803-834.

[3] J. J. Dongarra, and E. Grosse, "Distribution of mathematical software by electronic mail," Communications of the ACM, Vol. 30, No. 5, 1987.

[4] David M. Gay, "Electronic mail distribution of linear programming test problems," COAL newsletter \#13, December 1985, pp. 10-12.

[5] L. Lovász, and H. E. Scarf, "The generalized basis reduction algorithm," Cowles Foundation Discussion Paper No. 946, 1990, Yale University, New Haven, Connecticut.

[6] Gerhard Reinelt, "TSPLIB-A traveling salesman problem library," ORSA Journal on Computing, Vol. 30, No. 4, 1991. 